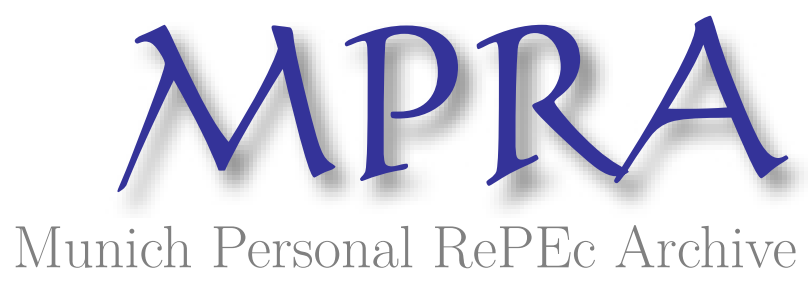

\title{
The optimal multi-stage contest
}

Fu, Qiang and Lu, Jingfeng

November 2006

Online at https://mpra.ub.uni-muenchen.de/946/

MPRA Paper No. 946, posted 28 Nov 2006 UTC 


\title{
The Optimal Multi-Stage Contest*
}

\author{
Qiang $\mathrm{Fu}^{\dagger}$ \\ National University of Singapore \\ Jingfeng $\mathrm{Lu}^{\ddagger}$ \\ National University of Singapore
}

April, 2006

\begin{abstract}
This paper investigates the optimal (effort-maximizing) structure of multi-stage sequentialelimination contests with pooling competition in each stage. We allow the contest organizer to design the contest structure in two arms: contest sequence (the number of stages, and the number of remaining contestants in each stage), and prize allocation. First, we find that the optimality of "winner-take-all" (single final winner, single final prize, no intermediate prizes) is independent of the contest sequence. Second, we show that the more complete the contest sequence is, the more efforts can be induced from the contestants. Therefore, the optimal contest eliminates one contestant at each stage until the finale, while a single winner takes over the entire prize purse. Our results not only rationalize various forms of multi-stage contests conducted in reality, such as the well-known Fox TV show "American Idol", but also shed light on the design of internal organizational hierarchy.
\end{abstract}

JEL Nos: C7, D7

\section{Introduction}

Situations in which economic agents expend costly and non-refundable resources in order to win a limited number of prizes are fairly ubiquitous. For instance, high school students engage in academic efforts to compete for college admissions. Firms participate in research

*We are especially grateful for the inspiring discussion with Kyung Hwan Baik. We thank Michael Baye, Ivan Png, Indranil Chakaraborty, Benny Moldovanu, Lixin Ye, Hongjun Zhong and Jimmy Chan, and all seminar participants at National University of Singapore, and Shanghai University of Finance and Economics for helpful comments and suggestions.

${ }^{\dagger}$ Qiang Fu: Department of Business Policy, National Univeristy of Singapore, 1 Business Link, SINGAPORE, 117592; Tel: (65)65163775, (65)67795059, bizfq@nus.edu.sg.

$\ddagger$ Jingfeng Lu: Department of Economics, National University of Singapore, 10 Kent Ridge Crescent, SINGAPORE, 119260; Tel: (65)65166026, Fax: (65)67752646, Email: ecsljf@nus.edu.sg. 
tournament to win a procurement contract of innovation. Cities lobby the International Olympic Committee in order to host Olympic Games. In modeling contests, the economic literature conventionally assumes that the contest lasts for only one stage, in which each contestant competes against all others for a single prize ("winner-take-all"). Many contests in reality, however, last for more than one stages, and require contestants to repeatedly exert efforts. In the final stage, the finalists expend resources to compete for the prizes; while in each preliminary stage, the remaining contestants compete for the "tickets" to the next round. In most of the contexts, no prize other than the "tickets" is awarded in the preliminary stages.

Numerous real world contest settings exhibit such a multi-phase sequential competition structure. One such example is the "election of London" to host the 2012 summer Olympic Game. While 9 cities initially submit applications, only 5 of them (London, Madrid, Moscow, New York and Paris) are shortlisted as the candidates for the final election. The 2005 DARPA (Defense Advanced Research Projects Agency) Grand Challenge, a race among autonomous robots held in the Mojave Desert along a route of 132.2 miles, also exemplifies a sequential competition setting. A total of 43 teams were selected out of 195 applicants to participate in the NQE (National Qualification Event), which cut the 43 teams down to 23 for the final race. In the end, "Stanley", the Stanford Racing Team's autonomous robotic car, completed the course first and earned a $\$ 2$ million prize. ${ }^{1}$ In research tournaments, the procurement firms select the few most attractive ideas from a larger pool of innovation proposals, and only the selected are eligible for the further race towards successful innovation (see Fullerton and McAfee, 1999). In many Asian countries, in contrast to the K-12 system in U.S., students have to take more than one major screening exams in order to be admitted into colleges. In recruiting new faculty members, economics departments usually interview a large group of candidates, but extend on-campus visit invitations to only a small number of them.

Central to the contest literature is the inquiry how the design or the rule of the contest affects the total efforts contestants expend. As argued by Gradstein and Konrad (1999), "... the contest structures are the outcome of a careful design with the view of attaining a variety of objectives, one of which is maximization of efforts by contenders". The efforts of the contestants benefit the contest organizer in many occasions, such as professional sports,

\footnotetext{
${ }^{1}$ We thank Ivan Png for alerting us of this example.
} 
research tournament, as well as political rent-seeking. Following this thread of thinking, our paper addresses the issue of optimal (effort-maximizing) contest design in a multi-phase sequential Tullock contest setting with pooling competition in each stage. We recognize that the structure of a multi-stage contest consists of two aspects: the sequence (the number of stages, and the number of remaining contestants in each stage), and the allocation of prizes. Specifically, we answer the following questions. First, given the sequence of a multi-stage contest, and a fixed total of prize purses, how does the contest organizer allocate the prize mass to the recipients in order to maximize the efforts? Does a "winnertake-all" (single-winner and single-prize) contest necessarily dominate a contest that awards intermediate prizes? Second, given the number of participants and the total value of prizes, does a multi-stage contest, which sequentially eliminates contestants, drive more efforts, as compared to a single-stage simultaneous contest? What is the optimal contest sequence? Third, does the sequence of the contest intertwine with the prize allocation in influencing the efforts?

We investigate the optimal contest sequence and the optimal prize allocation in a unified framework. We consider a multi-stage contest, in which $N$ identical contestants compete for a fixed prize mass, instead of a given single prize. Each stage-contest is a pooling contest, in which each remaining contestant competes against all other remaining contestants. In each preliminary stage, a contestant competes not only for the "tickets" to the next stage, but also for nonnegative intermediate prizes. In the final stage, the remaining contestants compete for nonnegative final prizes only. We allow the contest organizer to maximize the total efforts in two arms: choosing the optimal contest sequence and allocating optimally the prize mass. We first show that the optimal allocation of the prize mass is independent of the contest sequence structure, and a "winner-take-all" (single-prize) contest dominates all other prize allocations. We then show that the more "complete" the contest sequence is, the more efforts the contestants expend. ${ }^{2}$ As a consequence, the optimal contest that maximizes the total efforts is a $(N-1)$-stage "Pyramid" contest that eliminates one contestant at each stage, and a single final winner takes over the entire prize purse. To summarize, our study provides rationales for (i) the multi-stage contest widely observed in reality; and (ii) the winner-take-all principle commonly assumed in modeling rent-seeking competition in a more

\footnotetext{
${ }^{2}$ We will define the concept of completeness in Section 3.3.
} 
general setting.

\section{The Relation to the Literature}

Baye, Kovenock and de Vries (1993) raise the question "why do politicians frequently 'announce' that they have narrowed down a set of potential recipients of a 'prize' to a slate of finalists?" They examine the scenario, in which the politician optimally shortlists a set of finalists from a pool of rent seekers (who differ in their valuations for the final prize) to induce more competition, while the process of shortlisting does not involve rent-seeking activities on the part of contestants. A handful of papers, in contrast, have modeled the process of shortlisting as the preliminary stages of a sequential contest, which requires contestants to repeatedly expend efforts. For instance, Amegashie (1999) considers a two-stage contest. In the first stage of the contest, contestants are divided into groups, and a single winner stands out from each group to participate in the second-stage (final) competition.

Rosen (1986) models the organizational hierarchy as a series of pairwise contests: employees compete for promotion along the ladder of hierarchy. He shows that a larger top prize increases the overall efforts. Harbaugh and Klumpp (2005), as well as Matros (2005), consider two-stage tournament contests that group contestants in preliminary stages. In contrast to Amegashie (1999), they study the optimal intertemporal effort allocation of the contestants faced with resource constraints. Gradstein (1998) also contributes to this research agenda by comparing a simultaneous contest with a contest that consists of a series of pairwise subcontests.

Rosen (1986) considers a $2^{N}$-contestant $N$-stage sequential contest: in each stage, two of the remaining contestants are matched into head-to-head confrontation, and the winner survives for the next stage. Yet he does not justify the optimality of the contest structure. In the sense of endogenizing the contest structure, our paper is more closely related to Gradstein and Konrad (1999), as well as Moldovanu and Sela (2006). Gradstein and Konrad (1999) consider multi-stage imperfectly discriminatory contests that group identical contestants in preliminary stages. In contrast to Rosen (1986) and Amegashie (1999), they allow the contest organizer to flexibly design the contest structure as a matching scheme. They show that the multi-stage contest adopted by Rosen (1986) may emerge as the optimum if the contest success function is less discriminatory. In a perfectly discriminatory contest setting, 
Moldovanu and Sela (2006) study the optimal contest architecture that specifies whether and how the contestants should be split into subgroups in the first-stage competition. They find that the optimality of contest architecture depends upon the properties of contestants' cost function, as well as the objective of the contest organizer. A single grand static contest maximizes the expected total efforts for the case of linear cost of effort. If the effort cost function is convex, a designer can benefit from splitting the contestants into several subgroups in the preliminary stage of the contest.

Our paper differs from these papers in two regards. Firstly, we allow remaining contestants to compete against "all others" in each stage, instead of matching them into different groups. We consider each stage of competition in the sequential elimination process as a multiple-winner multiple-loser contest (as first suggested by the seminal paper of Clark and Riis (1996)): the winners are first selected to proceed to the next stage, while the rest of contestants are eliminated. ${ }^{3}$ Amegashie (2000) compares the two following ways of "shortlisting" in two-stage contests: pooling (contestants compete against all others in each stage) and grouping (contestants are divided into groups). He shows that the former generates a higher rent-dissipation rate. Fu and $\mathrm{Lu}$ (2005) also provide theoretical evidence supporting that pooling competition generates higher rent-dissipation rate. These studies partially justify why we adopt a pooling competition in each stage of the game. Our paper is also related to Fullerton and McAfee (1999) in modeling "shortlisting". In a two-stage model, they show that the optimal research tournament requires competing companies to participate in an all-pay auction (as a screening scheme) to win the entry, while only a subset of firms (the most competitive firms) engage in innovation activities.

Secondly, few of these papers allow for flexible prize allocation in multi-stage contest settings. Most of papers in the contest literature assume that the prize structure is exogenously given, and is governed by the "winner-take-all" principle: a single final winner receives a single indivisible prize. Besides Rosen (1986), the exceptions are Krishna and Morgan (1998), Moldovanu and Sela (2001) and Matros (2005). Krishna and Morgan (1998) justify the winner-take-all principle in small tournaments. Moldovanu and Sela (2001) con-

\footnotetext{
${ }^{3}$ Clark and Riis (1998) suggest an interesting multi-stage contest with a different rule. In their paper, winners are first selected in each stage to receive the stage prizes, but then they have to exit from the contest; while the losers proceed to the next stage and continue to exert efforts in order to compete for the remaining prizes.
} 
sider the optimal prize allocation in a one-stage perfectly discriminatory contest (all-pay auction). Matros (2005) allows the contest organizer to flexibly allocate his/her budget between a single winner's prize and losers' rewards to maximize the total efforts in a two-stage contest, and shows that a winner-take-all contest dominates.

Our paper proceeds as follows. Section 2 sets up the model. In Section 3, we first derive the general solution of equilibrium efforts in a multi-stage multi-prize contest. We then present our results on the optimal contest structure. In addition, we discuss the implications and applications of these results. Section 4 provides a concluding remark.

\section{The Model}

$N(\geq 3)$ risk-neutral contestants are engaged in a multi-stage sequential contest with nonnegative intermediate and final prizes. The contest organizer has a total budget of $\Gamma_{0}$ for prize allocation. For this moment, we fix the sequence of the contest, i.e. the number of stages and the numbers of survivors in each stage. Let $L$ denote the number of stages in the contest, and $N_{l}$ denote the number of contestants in stage $l \in\{1,2, \ldots, L\}$. In stage $l \in\{1,2, \ldots, L-1\}, N_{l}$ contestants participate, and $N_{l+1}$ of them survive and proceed to the next stage. Clearly, we have $N_{1} \equiv N$. There are $N_{l}$ nonnegative intermediate prizes $W_{l}^{m}, m \in\left\{1, \ldots, N_{l}\right\}$, available for each stage $l \in\{1,2, \ldots, L-1\}$. At stage $L, N_{L}$ contestants compete for $N_{L}$ nonnegative final prizes $W_{L}^{m}, m \in\left\{1, \ldots, N_{L}\right\} .{ }^{4}$ The sequence of a given contest is therefore represented by a $L$-term non-increasing sequence $\left\{N_{1}, N_{2}, \ldots, N_{L}\right\}$. Clearly, we should have $N_{1}=N \geq N_{2} \geq \ldots \geq N_{L} \geq 1$.

At all stages $l=1,2, \ldots, L$, the remaining contestants simultaneously exert their nonnegative efforts $e_{l}^{i}, i=1,2, \ldots, N_{l}$. The tickets to the next stage and the stage prizes are allocated in a sequential lottery process as modeled by Clark and Riis (1996). Once a contestant is selected, he/she is immediately removed from the pool, while the rest of them are eligible for the next draw. Define $\Omega_{l}^{m}$ to be the set of remaining contestants up for the $m$-th draw in stage $l$, where $m \in\left\{1,2, \ldots, N_{l}\right\}$. Denote $\left(e_{l}^{1}, \ldots, e_{l}^{i-1}, e_{l}^{i+1}, \ldots, e_{l}^{N_{l}}\right)$ by $\mathbf{e}_{l}^{-i}$. The conditional probability that a contestant $i \in \Omega_{l}^{m}$ is selected in the $m$-th draw is then given by

\footnotetext{
${ }^{4}$ Some final prizes are allowed to be zero.
} 


$$
p\left(e_{l}^{i}, \mathbf{e}_{l}^{-i} ; \Omega_{l}^{m}\right)=e_{l}^{i} / \sum_{j \in \Omega_{l}^{m}} e_{l}^{j} .
$$

In the case that all contestants up for a draw make zero effort, we assume that the selected is randomly chosen from the pool. Moreover, we assume that if $\Omega_{l}^{m}$ reduces to a singleton, then the only contestant is automatically selected for sure regardless of his effort. At stage $l \in\{1,2, \ldots, L\}$, the contestant selected in the $m$-th draw is awarded the prize $W_{l}^{m}$. In addition, at stage $l \in\{1,2, \ldots, L-1\}$, the contestants who are selected in the first $N_{l+1}$ draws proceed to the $(l+1)$-th stage, while the other $N_{l}-N_{l+1}$ contestants are eliminated. We define $\Gamma_{l} \equiv \sum_{m=1}^{N_{l}} W_{l}^{m}$ to be the sum of prizes awarded in stage $l$, and $\Gamma \equiv \sum_{l=1}^{L} \Gamma_{l}$ to be the total of the prizes in the whole contest.

Denote by $V_{l}$ the conditional (symmetric) equilibrium expected payoff of a representative contestant at stage $l$. For convenience, we define $V_{L+1}=0$. At stage $l \in\{1,2, \ldots, L\}$, a representative contestant $i$ rationally chooses his/her effort $e_{l}^{i}$ to maximize his expected payoff

$$
V_{l}^{i}=\sum_{m=1}^{N_{l+1}}\left[P_{m}\left(e_{l}^{i}, \mathbf{e}_{l}^{-i}\right)\left(V_{l+1}+W_{l}^{m}\right)\right]+\sum_{m=N_{l+1}+1}^{N_{l}}\left[P_{m}\left(e_{l}^{i}, \mathbf{e}_{l}^{-i}\right) W_{l}^{m}\right]-e_{l}^{i},
$$

where $P_{m}\left(e_{l}^{i}, \mathbf{e}_{l}^{-i}\right)$ is the probability that contestant $i$ is selected in the $m$-th draw. Note that $P_{m}\left(e_{l}^{i}, \mathbf{e}_{l}^{-i}\right)=\sum_{\forall \Omega_{l}^{m}}\left[\operatorname{Pr}\left(\Omega_{l}^{m}\right) \operatorname{Pr}\left(i \in \Omega_{l}^{m}\right) p\left(e_{l}^{i}, \mathbf{e}_{l}^{-i} ; \Omega_{l}^{m}\right)\right]$, where $\operatorname{Pr}\left(\Omega_{l}^{m}\right)$ is the probability that the remaining contestants up for the $m$-th draw are $\Omega_{l}^{m}$, and $\operatorname{Pr}\left(i \in \Omega_{l}^{m}\right)$ is the probability that contestant $i$ belongs to $\Omega_{l}^{m}$. Since we consider the symmetric equilibrium, we assume all contestants other than $i$ exert the same effort $e_{l}^{\prime}$ without loss of generality. Under this simplification,

$$
P_{m}\left(e_{l}^{i}, \mathbf{e}_{l}^{-i}\right)=\frac{\left(N_{l}-1\right) !}{\left(N_{l}-m\right) !}\left(\Pi_{k=1}^{m-1} \frac{e_{l}^{\prime}}{e_{l}^{i}+\left(N_{l}-k\right) e_{l}^{\prime}}\right) \frac{e_{l}^{i}}{e_{l}^{i}+\left(N_{l}-m\right) e_{l}^{\prime}} .
$$

Denote by $e_{l}$ the symmetric equilibrium effort. From (3), when $e_{l}^{i}=e_{l}, i=1, \ldots, N_{l}$, $\frac{\partial P_{m}\left(e_{l}^{i}, \mathbf{e}_{l}^{-i}\right)}{\partial e_{l}^{i}}$ is given by

$$
\frac{\partial P_{m}\left(e_{l}, \ldots, e_{l}\right)}{\partial e_{l}^{i}}=\frac{\left(1-\sum_{g=0}^{m-1} \frac{1}{N_{l}-g}\right)}{N_{l} e_{l}}
$$


The first order condition for the interior equilibrium effort $e_{l}$ is thus

$$
\sum_{m=1}^{N_{l+1}}\left[\frac{\partial P_{m}\left(e_{l}, \ldots, e_{l}\right)}{\partial e_{l}^{i}}\left(V_{l+1}+W_{l}^{m}\right)\right]+\sum_{m=N_{l+1}+1}^{N_{l}}\left[\frac{\partial P_{m}\left(e_{l}, \ldots, e_{l}\right)}{\partial e_{l}^{i}} W_{l}^{m}\right]-1=0 .
$$

(4) and (5) lead to

$$
\sum_{m=1}^{N_{l+1}}\left[\left(1-\sum_{g=0}^{m-1} \frac{1}{N_{l}-g}\right)\left(V_{l+1}+W_{l}^{m}\right)\right]+\sum_{m=N_{l+1}+1}^{N_{l}}\left[\left(1-\sum_{g=0}^{m-1} \frac{1}{N_{l}-g}\right) W_{l}^{m}\right]-N_{l} e_{l}=0 .
$$

Proposition 1 In a symmetric interior equilibrium of the contest in stage l, each remaining contestant exerts an effort of

$$
e_{l}=\frac{\sum_{m=1}^{N_{l+1}}\left[\left(1-\sum_{g=0}^{m-1} \frac{1}{N_{l}-g}\right)\left(V_{l+1}+W_{l}^{m}\right)\right]+\sum_{m=N_{l+1}+1}^{N_{l}}\left[\left(1-\sum_{g=0}^{m-1} \frac{1}{N_{l}-g}\right) W_{l}^{m}\right]}{N_{l}} .
$$

Proposition 1, which directly stems from the first order condition (6), gives the interior equilibrium individual effort of each remaining contestant in stage $l$. It strictly increases with the expected future payoff $V_{l+1}$ as the coefficient of $V_{l+1}$ is $\sum_{m=1}^{N_{l+1}}\left(1-\sum_{g=0}^{m-1} \frac{1}{N_{l}-g}\right)=$ $N_{l+1}-\sum_{g=0}^{N_{l+1}-1} \frac{N_{l+1}-g}{N_{l}-g} \geq 0$. Note that the term $\sum_{g=0}^{m-1} \frac{1}{N_{l}-g}$ strictly increases with $m$, the index for the order of the draw. Thus, the equilibrium effort $e_{l}$ also increases with the value of "earlier" prizes $W_{l}^{m}$ (the prizes awarded with earlier draws), where $m$ satisfies $1-\sum_{g=0}^{m-1} \frac{1}{N_{l}-g}>0$. However, $e_{l}$ decreases with the values of later prizes $W_{l}^{m}$, where $m$ satisfies $1-\sum_{g=0}^{m-1} \frac{1}{N_{l}-g}<0$

Define $\Phi_{l}$ to be $\Phi_{l} \equiv \sum_{m=1}^{N_{l+1}}\left[\left(1-\sum_{g=0}^{m-1} \frac{1}{N_{l}-g}\right)\left(V_{l+1}+W_{l}^{m}\right)\right]+\sum_{m=N_{l+1}+1}^{N_{l}}\left[\left(1-\sum_{g=0}^{m-1} \frac{1}{N_{l}-g}\right) W_{l}^{m}\right]$. The solution for the symmetric equilibrium effort is valid and satisfies (6) if and only if $\Phi_{l} \geq 0$ holds for $\left\{W_{l}^{m}\right\}_{m=1}^{N_{l}}$. Otherwise a corner solution applies and the equilibrium effort would be zero. $\Phi_{l}<0$ may happen when sufficiently large prizes are awarded for the latest draws. In this case, the contestants prefer not to make positive effort, but to wait for the latest prizes. As a result, the first order condition for interior solution fails, and the corner solution of zero effort arises. Here and hereafter, we assume $\Phi_{l} \geq 0$, and restrict our attention to the (unique) symmetric interior equilibrium. In Section 3.2, we will show in detail that there is no loss of generality to focus only on the prize allocations that lead to interior equilibrium. 
Rearrange the terms in (6), we have

$$
N_{l} e_{l}=N_{l+1} V_{l+1}-V_{l+1} \sum_{g=0}^{N_{l+1}-1} \frac{N_{l+1}-g}{N_{l}-g}+\Gamma_{l}-\sum_{m=1}^{N_{l}}\left(W_{l}^{m} \sum_{k=1}^{m} \frac{1}{N_{l}-k+1}\right) .
$$

Define the total efforts $E \equiv \sum_{l=1}^{L} N_{l} e_{l}$. In this context, we assume that the total efforts accrue to the benefit of the contest organizer. Thus, the contest organizer is to choose the optimal sequences $\left\{N_{l}\right\}_{l=1}^{L}$ and prize allocation $\left\{W_{l}^{m} \mid m=1, \ldots, N_{l} ; l=1, \ldots, L\right\}$ to maximize the total efforts $E$, subject to the budget constraint

$$
\Gamma \leq \Gamma_{0}
$$

\section{Results}

\subsection{Preliminary Results}

For convenience, we define $\Gamma_{L+1}=0$. In a stage $l, \forall l \in\{1,2, \ldots, L\}$, every symmetric contestant has the same chance of winning each component of the total stage-award $N_{l+1} V_{l+1}+\Gamma_{l}$ (including $N_{l}$ stage prizes and $N_{l+1}$ tickets to the next stage) in a symmetric equilibrium. Therefore, the conditional equilibrium expected payoff of a representative contestant at stage $l$ is $V_{l}=\left(N_{l+1} V_{l+1}+\Gamma_{l}\right) / N_{l}-e_{l}$, where $e_{l}$ is his/her equilibrium effort at stage $l$. The total of the $N_{l}$ contestants' equilibrium expected payoffs can then be written as

$$
N_{l} V_{l}=N_{l+1} V_{l+1}+\Gamma_{l}-N_{l} e_{l}
$$

which implies

$$
N_{l} e_{l}=N_{l+1} V_{l+1}+\Gamma_{l}-N_{l} V_{l} .
$$

Lemma $1 E=\Gamma-N V_{1}$.

Proof. Summing up (11) over the $L$ stages gives

$$
\begin{aligned}
E & \equiv \sum_{l=1}^{L-1} N_{l} e_{l}+N_{L} e_{L} \\
& =\sum_{l=1}^{L-1}\left(N_{l+1} V_{l+1}-N_{l} V_{l}\right)+\sum_{l=1}^{L-1} \Gamma_{l}+N_{L} e_{L} \\
& =N_{L} V_{L}-N V_{1}+\sum_{l=1}^{L-1} \Gamma_{l}+N_{L} e_{L} .
\end{aligned}
$$


Note that in the last stage of the contest, i.e. stage $L$, we have $N_{L} V_{L}=\Gamma_{L}-N_{L} e_{L}$. Thus,

$$
\begin{aligned}
E & =\Gamma_{L}-N V_{1}+\sum_{l=1}^{L-1} \Gamma_{l} \\
& =\Gamma-N V_{1} .
\end{aligned}
$$

\section{Q.E.D.}

Lemma 1 is fairly intuitive. $V_{1}$ represents the conditional expected payoff a representative contestant receives in the first stage. The contestant does not only gain from the intermediate prizes awarded in this stage, but also from the values of the "tickets" to future stages. As a consequence, $V_{1}$ is the payoff every contestant expects from the whole contest in the very beginning of the contest. Thus, $N V_{1}$ represents the total surplus all contestants receive in the contest, which, by the risk neutrality of the contestants, is equivalent to the difference between the total prize mass $\Gamma$ and the total efforts $E$.

From (8), the total equilibrium efforts in each stage are determined by the conditional expected payoffs of a representative contestant in next stage, and the stage prizes awarded in the current stage. Using (8) and (11), we can write in the following Lemma contestants' payoffs in the current stage $\left(V_{l}\right)$ in terms of the future expected payoff $\left(V_{l+1}\right)$, and the intermediate prizes of the current stage prizes.

Lemma $2 N_{l} V_{l}=V_{l+1} \sum_{g=0}^{N_{l+1}-1} \frac{N_{l+1}-g}{N_{l}-g}+\sum_{m=1}^{N_{l}}\left(W_{l}^{m} \sum_{k=1}^{m} \frac{1}{N_{l}-k+1}\right)$, for $l \in\{1,2, \ldots, L\}$.

Using Lemma 2, we are able to recursively solve for $V_{1}$ as in Lemma 3, which states that $V_{1}$ can be written as a function of the prizes in the current and all future stages.

Lemma $3 V_{1}=\sum_{l=1}^{L}\left\{\left(\Pi_{j=1}^{l} \frac{1}{N_{j}}\right)\left(\Pi_{j=1}^{l-1} \sum_{g=0}^{N_{j+1}-1} \frac{N_{j+1}-g}{N_{j}-g}\right) \sum_{m=1}^{N_{l}}\left(W_{l}^{m} \sum_{k=1}^{m} \frac{1}{N_{l}-k+1}\right)\right\}$.

Combining Lemmas 1 and 3, we have the following result.

Proposition 2 A N-person L-stage sequential-elimination contest, with the sequence structure $\left\{N_{l}\right\}_{l=1}^{L}$, prize allocation $\left\{W_{l}^{m} \mid m=1, \ldots, N_{l} ; l=1, \ldots, L\right\}$ and a total prize purse $\Gamma$, induces the total equilibrium efforts of

$$
E=\Gamma-N \sum_{l=1}^{L}\left\{\left(\Pi_{j=1}^{l} \frac{1}{N_{j}}\right)\left(\prod_{j=1}^{l-1} \sum_{g=0}^{N_{j+1}-1} \frac{N_{j+1}-g}{N_{j}-g}\right) \sum_{m=1}^{N_{l}}\left(W_{l}^{m} \sum_{k=1}^{m} \frac{1}{N_{l}-k+1}\right)\right\} .
$$




\subsection{The Optimal Prize Allocation}

Lemma 3 shows that given the sequence of the contest, a contestant's equilibrium surplus $V_{1}$ completely depends on the allocation of the prize purses. If the contest organizer intends to maximize the total efforts $E$, how many final winners should be allowed for? Does it pay to create intermediate prizes that are awarded in earlier stages? Next, we address how the contest organizer optimally allocates his/her total budget $\Gamma_{0}$ over the $\sum_{l=1}^{L} N_{l}$ possible prizes. By Lemma 1, the optimal prize allocation that maximizes the total efforts must minimize the equilibrium surplus $V_{1}$. Thus, we can focus on the minimization of $V_{1}$ to solve the original effort-maximization problem.

Before we proceed, we first clarify why there is no loss of generality if we focus on prize allocations that lead to symmetric interior equilibrium. Recall (6). An interior equilibrium requires $\Phi_{l} \equiv \sum_{m=1}^{N_{l+1}}\left[\left(1-\sum_{g=0}^{m-1} \frac{1}{N_{l}-g}\right)\left(V_{l+1}+W_{l}^{m}\right)\right]+\sum_{m=N_{l+1}+1}^{N_{l}}\left[\left(1-\sum_{g=0}^{m-1} \frac{1}{N_{l}-g}\right) W_{l}^{m}\right] \geq 0$. Suppose that the prize allocation makes $\Phi_{l}<0$, which leads to the corner solution, i.e. $e_{l}=0$. First, note that if we allocate the entire $\Gamma_{l}$ to the first draw prize, it must follow that $\Phi_{l} \geq 0$. Second, $\Phi_{l}$ is continuous in $\left\{W_{l}^{m}\right\}_{m=1}^{N_{l}}$. Thus, the contest organizer can always shift the prize mass $\Gamma_{l}$ from later prizes to earlier prizes within the stage, and reach an allocation that delivers exactly $\Phi_{l}=0$. The first order condition (6) is then reinstated and it renders an interior equilibrium with $e_{l}=0$. Thus, by appropriately shifting the prize mass from later prizes to earlier prizes, we can always apply condition (6) and obtain an interior equilibrium, which generates the same outcome as the corner solution equilibrium. Note from (10) that this adjustment of prizes does not alter $V_{l}$ as well. As a result, it does not affect the total efforts in any other stage of the contest. This means that we can ignore the possibility of a corner solution, and assume $\Phi_{l}$ to be nonnegative without loss of generality.

Theorem 1 ("Winner-take-all") Given a contest sequence $\left\{N_{l}\right\}_{l=1}^{L}$, the optimal contest prize allocation that maximizes the total efforts $E$ requires the entire prize purse to be allocated to the first prize in the final stage, i.e. $W_{L}^{1}=\Gamma_{0}$.

Proof. The proof consists of three steps.

Define for brevity $D_{l} \equiv\left(\Pi_{j=1}^{l} \frac{1}{N_{j}}\right)\left(\prod_{j=1}^{l-1} \sum_{g=0}^{N_{j+1}-1} \frac{N_{j+1}-g}{N_{j}-g}\right) \sum_{m=1}^{N_{l}}\left(W_{l}^{m} \sum_{k=1}^{m} \frac{1}{N_{l}-k+1}\right)$. From Lemma 
3 , we can then write $V_{1}$ as the sum of the $L$ separate terms $D_{l}, l \in\{1,2, \ldots, L\}$, i.e.,

$$
V_{1}=\sum_{l=1}^{L} D_{l}
$$

Step One: $W_{l}^{m}=0$, if $m>1, l \in\{1,2, \ldots, L\}$.

For any fixed $\Gamma_{l}, l \in\{1,2, \ldots, L\}, D_{l}$ is minimized if the entire prize purse in stage $l$ is allocated to the first prize $W_{l}^{1}$ because $\sum_{k=1}^{m} \frac{1}{N_{l}-k+1}$ increases with $m$. Thus for the optimal prize allocation, we must have $W_{l}^{m}=0$, if $m>1, l \in\{1,2, \ldots, L\}$.

Step Two: $W_{l}^{1}=0$, for $l \in\{1,2, \ldots, L-1\}$, and $W_{L}^{1}=\Gamma$, given a fixed total prize purse $\Gamma \leq \Gamma_{0}$.

Step one implies that $V_{1}$ can be reduced to the following form for the optimal prize allocation

$$
V_{1}=\sum_{l=1}^{L}\left\{\left(\Pi_{j=1}^{l} \frac{1}{N_{j}}\right)\left(\Pi_{j=1}^{l-1} \sum_{g=0}^{N_{j+1}-1} \frac{N_{j+1}-g}{N_{j}-g}\right) \frac{W_{l}^{1}}{N_{l}}\right\} .
$$

Next, we show that for $l \in\{1,2, \ldots, L-1\}$, if we move the prize mass $W_{l}^{1}$ to $W_{l+1}^{1}$, it further reduces $V_{1}$. For this purpose, we need only to compare the coefficients of $W_{l}^{1}$ and $W_{l+1}^{1}$. Thus, we compare $\left(\Pi_{j=1}^{l} \frac{1}{N_{j}}\right)\left(\Pi_{j=1}^{l-1} \sum_{g=0}^{N_{j+1}-1} \frac{N_{j+1}-g}{N_{j}-g}\right) \frac{1}{N_{l}}$ with $\left(\Pi_{j=1}^{l+1} \frac{1}{N_{j}}\right)\left(\Pi_{j=1}^{l} \sum_{g=0}^{N_{j+1}-1} \frac{N_{j+1}-g}{N_{j}-g}\right) \frac{1}{N_{l+1}}$. Ignoring the common elements, we only need to compare $\frac{1}{N_{l}}$ to $\left(\frac{1}{N_{l+1}}\right)^{2} \sum_{g=0}^{N_{l+1}-1} \frac{N_{l+1}-g}{N_{l}-g}$. Obviously, $\left(\frac{1}{N_{l+1}}\right)^{2}$ $\sum_{g=0}^{N_{l+1}-1} \frac{N_{l+1}-g}{N_{l}-g}<\left(\frac{1}{N_{l+1}}\right)^{2}\left(N_{l+1} \frac{N_{l+1}}{N_{l}}\right)=\frac{1}{N_{l}}$, because, $\frac{N_{l+1}-g}{N_{l}-g}<\frac{N_{l+1}}{N_{l}}$ if $g>0$. It follows that the weight on $W_{l}^{1}$ is strictly greater than that on $W_{l+1}^{1}$, which implies that shifting the mass of $W_{l}^{1}$ to $W_{l+1}^{1}$ strictly reduces $V_{1}$.

Thus in order to minimize $V_{1}$, all prize mass allocated to prizes in an earlier stage $(l<L)$ should be reallocated to $W_{L}^{1}$. Given the total amount of prizes $\Gamma$, we thus have $W_{L}^{1}=\Gamma$. Thus, we show that a "winner-take-all" contest maximizes the total efforts for any given contest sequence.

Step Three: $\Gamma=\Gamma_{0}$, i.e., the contest organizer uses up the entire budget on prizes.

Based on steps one and two, Proposition 2 leads to that $E$ can be reduced to the following form for the optimal prize allocation

$$
E=\Gamma\left[1-N\left(\Pi_{j=1}^{L} \frac{1}{N_{j}}\right)\left(\Pi_{j=1}^{L-1} \sum_{g=0}^{N_{j+1}-1} \frac{N_{j+1}-g}{N_{j}-g}\right) \frac{1}{N_{L}}\right]
$$


Note that $N\left(\Pi_{j=1}^{L} \frac{1}{N_{j}}\right)\left(\Pi_{j=1}^{L-1} \sum_{g=0}^{N_{j+1}-1} \frac{N_{j+1}-g}{N_{j}-g}\right) \frac{1}{N_{L}}=\frac{N}{N_{L}^{2}} \Pi_{j=1}^{L-1}\left[\frac{1}{N_{j}} \sum_{g=0}^{N_{j+1}-1} \frac{N_{j+1}-g}{N_{j}-g}\right]<\frac{N}{N_{L}^{2}} \Pi_{j=1}^{L-1} \frac{N_{j+1}}{N_{j}}=$ $\frac{1}{N_{L}} \leq 1$. Thus $E$ strictly increases with $\Gamma$. Therefore, the entire budget $\Gamma_{0}$ should be allocated to $W_{L}^{1}$.

\section{Q.E.D.}

Theorem 1 establishes that if the contestant organizer has the flexibility to allocate a fixed prize mass, a multi-stage contest that maximizes the total efforts must combine all the resource into a single final prize and reward it to a single final winner, regardless of the sequence of the contest. Our results therefore provide a rationale for the commonly assumed "winner-take-all" principle in modeling rent-seeking competition. Clark and Riis (1996) show that contestants expend more efforts if the contest is governed by a "winner-take-all" rule than they do if the number of positive prizes exceeds one. Theorem 1 confirms this insight in the context of multi-stage elimination contests.

Lemma 3 shows that $V_{1}$ is a weighted sum of the prizes awarded in all stages. The weights on prizes exhibit two interesting features. On one hand, within a single stage, an earlier prize has a smaller weight. This is due to the fact that a higher probability of winning an earlier prize demands higher efforts from a contestant. As a result, within a stage $l$, the contest organizer can increase the efforts by allocating the entire stage purse $\Gamma_{l}$ to the firstdraw prize, i.e. $W_{l}^{1}=\Gamma_{l}{ }^{5}$ Therefore, we can focus on the contest structure that allocates the prize purse only to the first-draw prize of each stage. On the other hand, between any two stages, a first-draw prize awarded in a later stage has a smaller weight. The intuition is that contestants have to (repeatedly) exert their further efforts to win a prize awarded in a later stage. In other words, a prize at a higher rank of the ladder demands more efforts from a contestant. To induce the highest subsequent efforts, all resources should then be allocated as one single prize at the last stage. Aggregating both the "within" and "between" effects leads to the optimality of "winner-take-all" in our multi-stage setting.

\subsection{The Optimal Contest Sequence: Pyramid Contest}

Having established the "winner-take-all" principle as the optimal prize allocation rule in any multi-stage contest, it remains to ask what is the optimal sequence of the contest (the number of stages and the number of remaining contestants in each stage). Next, we study

\footnotetext{
${ }^{5}$ This effect is consistent with the "winner-take-all" principle in Moldovanu and Sela (2001).
} 
how the contest organizer optimally chooses the sequence $\left\{N_{l}\right\}_{l=1}^{L}$ to maximize the total efforts $E$. By Theorem 1, the allocation of prize mass is independent of the sequence of the contest, we therefore simply restrict our attention to the case with a single prize $W_{L}^{1}=\Gamma_{0}$.

Rewriting Lemma 3 by setting all prizes other than $W_{L}^{1}$ to be zero leads to

$$
V_{1}=\left(\Pi_{j=1}^{L} \frac{1}{N_{j}}\right)\left(\Pi_{j=1}^{L-1} \sum_{g=0}^{N_{j+1}-1} \frac{N_{j+1}-g}{N_{j}-g}\right) \frac{\Gamma_{0}}{N_{L}} .
$$

First of all, for mathematical convenience, we consider only the contest sequences with $N_{L}=1$ for the optimal sequence without loss of generality. By the optimality of "winnertake-all" principle in prize allocation, a single winner survives the last stage of the contest and takes over the entire prize purse. Thus, a $L$-stage contest with $N_{L}>1$ is equivalent to a hypothetical $(L+1)$-stage contest represented by the sequence $\left\{\left\{N_{l}\right\}_{l=1}^{L}, 1\right\}$, i.e. $N_{L+1}=1$. In the hypothetical $(L+1)$-stage contest, one contestant is selected at stage $L$ to enter stage $L+1$, but does not receive prize in stage $L$. But the "last man standing" automatically wins $\Gamma_{0}$ in stage $L+1$ without exerting effort. Consequently, we have $e_{L+1}=0$ and $V_{L}=\Gamma_{0}$, and equation (18) still applies. We thus consider in the following analysis only contest sequences with $N_{L}=1$.

Second, we assume that the contest sequence $\left\{N_{l}\right\}_{l=1}^{L}$ is strictly decreasing. Equation (16) implies that if $N_{l}=N_{l+1}$, then eliminating stage $l$ does not affect $V_{1}$. Based on the above two results, we can search for the optimal contest sequence by considering only the sequences $\left\{N_{l}\right\}_{l=1}^{L}$, where $N_{1}=N>N_{2}>\ldots>N_{L}=1$, without loss of generality.

In a $L$-stage contest with a sequence of $\left\{N_{l}\right\}_{l=1}^{L}$ where $N_{L}=1$, suppose there exists $J<L$ such that $N_{J}-N_{J+1}>1$. We imagine to insert an additional stage between stage $J$ and stage $J+1$, in which $M \in\left\{N_{J+1}+1, \ldots, N_{J}-1\right\}$ contestants selected from the $N_{J}$ contestants at the $J$-th stage compete for the $N_{J+1}$ tickets to stage $J+1$. Does adding this additional stage necessarily induce more total efforts from the contestants?

The effect of this additional stage on the total efforts in the contest is not readily seen: although the additional stage $M$ creates a new source of efforts $M e_{M}$, the impact of this additional stage on the contestants' effort entries in previous stages is ambiguous. First, from (8), $N_{J} e_{J}$ may either decrease or increase, although $N_{J} e_{J}$ tends to be reduced as the value of the ticket to the next stage is lower $\left(V_{M}=\left(N_{J+1} V_{J+1}-M e_{M}\right) / M<V_{J+1}\right)$. This is due to the fact that in (8), $N_{J} e_{J}$ is not a monotonic function of the number of survivors to 
the next stage. ${ }^{6}$ For the same reason, the impact of the additional stage on the stage payoff $V_{J}$ is also indefinite. Second, the additional stage's impact on the efforts in stages prior to stage $J, N_{j} e_{j}, j<J$, is also ambiguous. From (8), $N_{j} e_{j}, j<J$, would change in the same direction as $V_{j+1}$, which in turn changes in the same direction as $V_{J}$.

Let $\mathcal{E}\left(\left\{N_{l}\right\}\right)$ denote the set composed of all the integers in the sequence $\left\{N_{l}\right\}$.

Definition 1 A contest sequence $\left\{\tilde{N}_{l}\right\}$ is more complete than $\left\{N_{l}\right\}$ if and only if $\mathcal{E}\left(\left\{N_{l}\right\}\right) \subset$ $\mathcal{E}\left(\left\{\tilde{N}_{l}\right\}\right)$.

We show in the following theorem that any additional stage always increases the total efforts, regardless of the existing contest structure.

Theorem 2 The more complete the contest sequence is, the higher the total efforts are induced.

Proof. Denote by $E_{0}$ the total efforts in the original contest $\left\{N_{l}\right\}$, while by $E_{M}$ the total efforts in the new contest after one additional stage $M$ is inserted. We only need to show $E_{M}>E_{0}$.

Denote by $\widetilde{V}_{1}$ the equilibrium expected payoff that the $N$ contestants anticipate at the first stage of the contest after the additional stage is inserted. By Lemma 1, we simply need to show $\widetilde{V}_{1}<V_{1}$.

Under the optimal prize allocation characterized in Theorem 1, Lemma 2 leads to that

$$
V_{1}=\frac{V_{J+1}}{\prod_{l=1}^{J} N_{l}} \cdot \prod_{l=1}^{J}\left(\sum_{g=0}^{N_{l+1}-1} \frac{N_{l+1}-g}{N_{l}-g}\right)
$$

Similarly,

$$
\widetilde{V}_{1}=\frac{V_{J+1}}{M \prod_{l=1}^{J} N_{l}} \cdot\left[\prod_{l=1}^{J-1}\left(\sum_{g=0}^{N_{l+1}-1} \frac{N_{l+1}-g}{N_{l}-g}\right)\right]\left(\sum_{g=0}^{M-1} \frac{M-g}{N_{J}-g}\right)\left(\sum_{g=0}^{N_{J+1}-1} \frac{N_{J+1}-g}{M-g}\right) .
$$

\footnotetext{
${ }^{6}$ The following example shows that the component $N_{l+1}-\sum_{g=0}^{N_{l+1}-1} \frac{N_{l+1}-g}{N_{l}-g}$ is not a monotonic function of $N_{l+1}$. Assume $N_{l}=5$. Then $N_{l+1}-\sum_{g=0}^{N_{l+1}-1} \frac{N_{l+1}-g}{N_{l}-g}=1.35$ when $N_{l+1}=2 ; N_{l+1}-\sum_{g=0}^{N_{l+1}-1} \frac{N_{l+1}-g}{N_{l}-g}=1.57$ when $N_{l+1}=3 ; N_{l+1}-\sum_{g=0}^{N_{l+1}-1} \frac{N_{l+1}-g}{N_{l}-g}=1.28$ when $N_{l+1}=4$.
} 
To establish that $\widetilde{V}_{1}<V_{1}$, we need to show $\frac{1}{M}\left(\sum_{g=0}^{N_{J+1}-1} \frac{N_{J+1}-g}{M-g}\right)\left(\sum_{g=0}^{M-1} \frac{M-g}{N_{J}-g}\right)<\sum_{g=0}^{N_{J+1}-1} \frac{N_{J+1}-g}{N_{J}-g}$. Since $\frac{M-g}{N_{J}-g}$ is decreasing in $g, \frac{\sum_{g=0}^{M-1} \frac{M-g}{N_{J}-g}}{M}<\frac{\sum_{g=0}^{N_{J+1}-1} \frac{M-g}{N_{J}-g}}{N_{J+1}}$. Thus,

$$
\begin{aligned}
& \frac{1}{M}\left(\sum_{g=0}^{N_{J+1}-1} \frac{N_{J+1}-g}{M-g}\right)\left(\sum_{g=0}^{M-1} \frac{M-g}{N_{J}-g}\right) \\
< & \frac{1}{N_{J+1}}\left(\sum_{g=0}^{N_{J+1}-1} \frac{N_{J+1}-g}{M-g}\right)\left(\sum_{g=0}^{N_{J+1}-1} \frac{M-g}{N_{J}-g}\right) \\
< & \sum_{g=0}^{N_{J+1}-1} \frac{N_{J+1}-g}{N_{J}-g} .
\end{aligned}
$$

The last step follows Chebyshev Sum Inequality.

\section{Q.E.D.}

Theorem 2 is important. It establishes that an additional stage of competition always reduces a representative contestant's expected payoff $V_{1}$, so as to increase the total efforts. A contest sequence is not optimal, as long as the difference between any two successive terms in the sequence $\left\{N_{l}\right\}_{l=1}^{L}$ exceeds one. The total efforts of the contest can be increased if additional stages can be inserted, regardless of its existing structure. Thus, the optimal contest sequence is represented by a $N$-term strictly decreasing arithmetic sequence $\left\{N_{l} \mid N_{l}=N-l+1, l=1,2, \ldots, N.\right\}$. The last term $N_{N}=1$ represents the unique final winner. In other words, the contest lasts for $N-1$ stage, and one contestant is eliminated in each stage. ${ }^{7}$ We name it as a complete-sequence "Pyramid" contest.

Theorem 3 In a setting with $N$ contestants and a prize budget $\Gamma_{0}$, the effort-maximizing sequential contest with pooling competition in each stage lasts for $N-1$ stages, while eliminating one contestant each stage, and a single final winner takes over the entire prize purse of $\Gamma_{0}$.

Theorem 3 naturally stems from Theorem 1 and Theorem 2. Thus, we conclude that the optimal contest must be organized as a "winner-take-all" complete-sequence "Pyramid" contest.

Theorem 4 The optimally designed $N$-person contest with the total prize purse of $\Gamma_{0}$, i.e.

\footnotetext{
${ }^{7}$ In stage $N-1$, two remaining contestants compete for one final prize.
} 
the "winner-take-all" "Pyramid" contest, induces a total equilibrium effort of

$$
E=\Gamma_{0} \cdot\left\{1-\frac{\prod_{l=1}^{N-1}\left[\sum_{g=0}^{N-l-1} \frac{(N-l)-g}{(N-l+1)-g}\right]}{\prod_{l=1}^{N-1}(N-l+1)}\right\} .
$$

Theorem 4 explicitly shows the equilibrium total efforts in the optimally designed $N$ person contest. The result directly arises from Lemma 1 and (18), and the fact that the sequence structure of the optimal contest is represented by a complete sequence of integers from $N$ to 1 .

\subsection{Discussion}

So far we have shown that a contest drives more efforts, provided that the contestants have to survive a longer line of shots before they win the final prize. Our results therefore provide a rationale for the widely observed multi-phase sequential competition in reality. Baye, Kovenock, and de Vries (1993) argue that the contest organizer (politicians) may strategically shortlist a subset of finalists, according to their valuations, to participate in rent-seeking competitions (for instance, IOC selects potential candidate cities up for the election of hosting cities of Olympic Games), in order to increase the rent-seeking revenue. We suggest an alternative view to this thread of thinking: shortlisting benefits the contest organizer even if the contestants are identical.

Our paper provides useful insights to contests design. We show that the optimal contest that generates the most efforts is a "winner-take-all" complete-sequence "Pyramid" contest. The famous Fox TV show "American Idol" echoes our results pretty well. The show is basically a singing contest. Twelve contestants are picked out from "thousands and thousands of hopeful superstars". These twelve "winners", however, are not met with immediate success. The remaining part of the contest proceeds exactly in the form of a "Pyramid" contest which we have established to be optimal. The series of the shows then last for eleven weeks. In each week every remaining contestant makes his/her performance. After each show, one of the remaining contestants is voted off by viewers, while the others proceed to the next stage. The procedure repeats until the finale, in which two survivors compete face to face, and one of them becomes the new "American Idol". We see that the organization 
structure of "American Idol" turns out to coincide with the effort-maximizing contest we have established.

Our results provide insights to contests design even when the organizer is faced with constraints on contest structure. For instance, a contest may have to be conducted with a fixed number of stages, while the contest organizer has only the flexibility to choose the number of contestants who survive each stage. Proposition 2 has fully characterized the maximal total efforts that result from any given contest sequence structure. The restricted optimal contest sequence can thus be identified through direct comparison across finite possibilities.

Rosen (1986) and Gradstein and Konrad (1999) draw the analogy between the internal hierarchy of an organization and a multi-stage contest. As argued by Gradstein and Konrad (1999): "One can interpret the organization's hierarchy in a steady state as consisting of a series of contests among the individual members of each level of the hierarchy for the promotion to an upper level." Our results have two important implications on the design of internal organizational structure. Firstly, we show, by Theorem 2, that a hierarchical structure does increase the total efforts agents expend inside the organization. This result provides an alternative rationale for organizational hierarchy. Secondly, our results shed light on the design of internal incentive (compensation) scheme. We show that in the optimal contest, the total of prize purses should be combined into a single final prize, and no intermediate award should be given away. In the context of organizational hierarchy, the winner-take-all principle may not be feasible. Yet our results do not lose its appeal in this aspect. Recall Lemma 3, which gives a representative contestant's expected payoffs in terms of the prize structure. We see that $V_{1}$ is in fact a weighted sum over the prizes, and the weights associated with the prizes diminish as the level of the prizes ascends. It implies that more generous purses for top-ranking prizes (wage, or other benefits) "maintain the incentive in career" (Rosen (1986)) and increase the overall efforts. Our results confirm the insights suggested by Rosen (1986): "contestants who succeed in attaining high ranks in elimination career ladders rest on their laurels in attempting to climb higher, unless top-ranking prizes are given a disproportionate weight in the purse". 


\section{Concluding Remarks}

This paper studies the optimal contest structure in a multi-phase sequential competition setting. We allow the contest organizer to design the optimal contest in two arms: the contest sequence and the allocation of a fixed total of prize purses. We show that the contest organizer must allocate the entire prize mass to a single final prize, regardless of the contest sequence. We further show that additional stages always increase the total efforts. This result provides important insights for the design of multi-stage contests. Therefore we conclude that the optimal contest must be a "winner-take-all" complete-sequence "Pyramid" contest that eliminates one contestant in each stage until the finale.

This paper concerns itself with one aspect of contests design: the maximization of total efforts, and leaves tremendous room for future extensions. First of all, we have not considered the cost of organizing the contest. The contest organizer may be concerned about the additional costs that could arise from additional stages, which should be taken into account in future research on the optimal design of multi-stage contests. Secondly, we do not consider the heterogeneity in abilities or preferences among contestants. One interesting extension is to allow for contestants with differing types. We believe that extensions in this direction will not vary the main themes of our results. Nevertheless, it is still interesting to investigate whether a stronger contestant is more likely to win the final prize in a multi-stage contest than he/she does in a single-stage one. In that sense, a model with asymmetric players may shed light on the screening effect of the multi-stage contest. In addition, our study considers a contest success function with linear impact of effort. Perhaps, another challenging extension would be to allow for other forms of contest technologies. Finally, our model assumes that contestants' efforts affect only the outcome of the sub-contest in the current stage. One may extend this model by allowing for "accumulatable" efforts, in which case efforts made in the current stage can be carried over into future stages and continue to influence contestants' likelihoods of winning. 


\section{References}

[1] Amegashie, J.A., The Design of Rent-Seeking Competitions: Committees, Preliminary and Final Contest, Public Choice, 1999, 99, 63-76.

[2] Amegashie, J.A., Some Results on Rent-Seeking Contests with Shortlisting, Public Choice, 2000, 105, 245-253.

[3] Baye, M.R., Kovenock, D., and de Vries, C.G., Rigging the Lobbying Process: An Application of All-Pay Auction, American Economic Review, 1993, 86, 289-293.

[4] Clark, D.J., and Riis, C., A Multiple-Winner Nested Rent-Seeking Contest, Public Choice, 1996, 87, 177-184.

[5] Clark, D.J., and Riis, C., Influence and the Discretionary Allocation of Several Prizes, European Journal of Political Economy, 1998, 14, 605-625.

[6] Fu, Q., and Lu, J., Replicating Contests: Revisited, working paper, 2005, National University of Singapore

[7] Fullerton, R.L., and McAfee, P.R., Auctioning Entry into Tournaments, Journal of Political Economy, 1999, 107, 573-605.

[8] Gradstein, M., Optimal Contest Design: Volume and Timing of Rent-Seeking in Contests, European Journal of Political Economy, 1998, 14, 575-585.

[9] Gradstein, M., and Konrad, K.A., Orchestrating Rent Seeking Contests, Economic Journal, 1999, 109, 535-545.

[10] Harbaugh, R., and Klumpp, T., Early Round Upsets and Championship Blowouts, Economic Inquiry, 2005, 43, 316-329.

[11] Krishna, V., and Morgan, J., The Winner-Take-All Principles in Small Tournaments, in Advances in Applied Microeconomics, M. Baye, ed., JAI Press, Stamford, 1998.

[12] Matros, A., Elimination Tournaments where Players Have Fixed Resources, 2005, working paper, University of Pittsburgh. 
[13] Moldovanu, B., and Sela, A., The Optimal Allocation of Prizes in Contests, American Economic Review, 2001, 91, 542-558.

[14] Moldovanu, B., and Sela, A., Contest Architecture, Journal of Economic Theory, 2006, $126,70-96$.

[15] Rosen, S., Prizes and Incentives in Elimination Tournaments, American Economic Review, 1986, 76, 701-715. 